\title{
A Comparison of Data Sources for Motor Vehicle Crash Characteristic Accuracy
}

\author{
Robert J. Grant, MD, MARY ANn Gregor, MHSA, PAUl W. BecK, MD, \\ RONALD F. MAIO, DO, MS
}

\begin{abstract}
Objective: To determine the accuracy of police reports (PRs), ambulance reports (ARs), and emergency department records (EDRs) in describing motor vehicle crash (MVC) characteristics when compared with an investigation performed by an experienced crash investigator trained in impact biomechanics. Methods: This was a cross-sectional, observational study. Ninety-one patients transported by ambulance to a university emergency department (ED) directly from the scene of an MVC from August 1997 to April 1998 were enrolled. Potential patients were identified from the ED log and consent was obtained to investigate the crash vehicle. Data describing MVC characteristics were abstracted from the $\mathrm{PR}, \mathrm{AR}$, and medical record. Variables of interest included restraint use (RU), air bag deployment (AD), and type of impact (TI). Agreements between the variables and the independent crash investigation were compared using kappa. Interrater reliability was determined using kappa by comparing a random sam-
\end{abstract}

ple of 20 abstracted reports for each data source with the originally abstracted data. Results: Agreement using kappa between the crash investigation and each data source was $0.588(95 \% \mathrm{CI}=0.508$ to 0.667$)$ for the PR, 0.330 (95\% CI = 0.252 to 0.407$)$ for the $\mathrm{AR}$, and $0.492(95 \% \mathrm{CI}=0.413$ to 0.572$)$ for the EDR. Variable agreement was $0.239(95 \% \mathrm{CI}=0.164$ to 0.314 ) for $\mathrm{RU}, 0.350$ (95\% CI $=0.268$ to 0.432 ) for $\mathrm{AD}$, and $0.631(95 \%=0.563$ to 0.698$)$ for TI. Interrater reliability was excellent (kappa $>0.8$ ) for all data sources. Conclusions: The strength of the agreement between the independent crash investigation and the data sources that were measured by kappa was fair to moderate, indicating inaccuracies. This presents ramifications for researchers and necessitates consideration of the validity and accuracy of crash characteristics contained in these data sources. Key words: injury control; motor vehicle crash; trauma registry; data sources. ACADEMIC EMERGENCY MEDICINE 2000; 7:892-897
$\mathrm{D}$ ESCRIPTIVE information about motor vehicle crashes (MVCs) is important and can impact acute patient care, trauma triage, and research. However, whether the data accurately describe MVC characteristics is an important consideration. Whether data are being used at the patient level or as part of a population-based study, it is necessary to assess whether inaccurate or missing data could affect outcomes. Three data sources are typically used to analyze MVC char-

From the Department of Traumatology and Emergency Medicine, University of Connecticut School of Medicine, Farmington, CT (RJG); University of Michigan Injury Research Center and Department of Emergency Medicine, University of Michigan, Ann Arbor, MI (MAG, RFM); and Department of Emergency Medicine, University of Pittsburgh School of Medicine, Pittsburgh, PA (PWB). All authors were affiliated with the University of Michigan when the study was conducted.

Received November 12, 1999; revision received March 6, 2000; accepted March 20, 2000. Presented at the SAEM annual meeting, Boston, MA, May 1999.

Supported by the University of Michigan Emergency Medicine Residency Research Fund.

Address for correspondence and reprints: Robert J. Grant, MD, Department of Trauma/EM, Hartford Hospital, 80 Seymour Street, Hartford, CT 06102. Fax: 860-545-1461; e-mail: rgrant@harthosp.org acteristics: the police report (PR) completed by the investigating officer that describes the drivers and vehicles involved; the ambulance report (AR) completed by the paramedic that describes information about the crash as well as care provided to the patient; and the emergency department record (EDR) completed by hospital staff that describes patientlevel crash information as well as care provided to the patient.

Previously, we completed a study detailing the variability of crash characteristics among data sources. ${ }^{1}$ To the best of our knowledge, this was the only previous study to make these comparisons. In this retrospective study, we compared the PR, AR, and EDR with an independent crash investigation that had been completed by a trained crash investigator. The findings suggested inaccuracies in the data sources reviewed, despite the limitations of the study that included a retrospective focus and a limit on the type of MVCs (crashes involving a newer model vehicle with airbag deployment in one of the vehicles).

Thus, to further test our hypothesis, we compared the data sources using a prospective study design and without a restriction on the type of crashes to be included. The data sources remained 
the same, and minor modifications were made to the crash characteristics of interest (Fig. 1). We hypothesized there would be substantial variability among the data sources when analyzing the crash characteristics.

\section{MeTHODS}

Study Design. This was a cross-sectional, observational study conducted in the emergency department (ED) of a university hospital with an emergency medicine residency program. This study was approved by the Institutional Review Board at the University of Michigan.

Study Setting and Population. The ED has an annual census of 52,000 and is verified as a Level 1 trauma center by the American College of Surgeons. The ED serves a suburban/rural county with a population of 290,000. Ambulance transports within the county are handled by a single provider and staffed by paramedics. The study population comprised MVC victims, both drivers and passengers, transported from the crash scene by ambulance to the ED during the period August 1997 to April 1998.

Study Protocol. Motor vehicle crash patients transported to the ED by ambulance were identified from the ED log. All patients transported by ambulance from the scene of an MVC were considered eligible for enrollment. Initially, every third eligible patient was selected for enrollment, and then contacted by phone to obtain consent. When more than one patient presented at a time from a crash scene, the ED arrival time was used for enrollment. However, after one month, every other eligible patient was selected for enrollment, and then contacted by phone, a protocol that continued for six months. During the last two months of the study, every eligible patient was enrolled to obtain the desired number of subjects. The sample size of 80 patients was estimated to yield $95 \%$ confidence intervals (CIs) ranging $\pm 10 \%$ from the estimated frequency for the categorical variables studied.

Demographic information was recorded for all eligible MVC patients. Consent was obtained via telephone to participate in the study and for the crash investigator to view the vehicle involved. The crash investigation coordinator from the University of Michigan Transportation Research Institute (UMTRI) examined the crash vehicle. The investigation, considered an industry standard, involved studying the crash vehicle and analyzing occupant interactions with the vehicle components and restraint systems. The crash investigator made determinations about safety belt use, air bag deployment, and type of impact during his investigation.
Study Code:

Data Source:

$$
\begin{aligned}
& \text { 1. Restraint Use } \\
& 1 \text { = Unrestrained } \\
& 2=\text { Restrained } \\
& 3=\text { Shoulder Belt Only } \\
& 4 \text { = Lap Belt Only } \\
& 5=\text { Child Safety Seat } \\
& 9=\text { Unknown } \\
& \text { 2. Air Bag Deployment } \\
& 1=\text { Deployed } \\
& 2=\text { Not Deployed/Not Equipped } \\
& 9=\text { Unknown } \\
& \text { 3. Type of Impact } \\
& 1=\text { Front End Impact } \\
& 2=\text { Driver Side Impact } \\
& 3=\text { Passenger Side Impact } \\
& 4=\text { Rear Impact } \\
& 5=\text { Rollover } \\
& 6=\text { Undercarriage } \\
& 9=\text { Unknown }
\end{aligned}
$$

Figure 1. Crash characteristics.

To minimize inter- and intrarater variability, the same investigator examined every vehicle.

Hard copies of the reports, the PR, AR, and EDR, were obtained from the respective sources. The PR is a standard form (UD-10) used by all law enforcement agencies in Michigan to document MVCs. This optically scanned form is completed by the investigating officer based on information obtained at the scene of the crash, and includes a crash diagram. The AR is a standard form used by paramedics and is a written, narrative report documenting history and procedures. The ambulance crew is required to complete the ambulance run report prior to leaving the hospital. The EDR may contain a number of forms depending on the level of care the patient receives. These forms include a trauma flow sheet, a narrative nurse note, and an emergency physician dictation. The trauma flow sheet was considered the primary data source for the EDR since the form had "check boxes" for the crash characteristics of interest. If data were unknown or missing, one of the other two sources was used and in the case of a discrepancy between data sources, the trauma flow sheet was used. Anatom- 
TABLE 1. Demographics of the Subjects

\begin{tabular}{|c|c|c|}
\hline & $\begin{array}{c}\text { Enrolled } \\
(n=91)\end{array}$ & $\begin{array}{l}\text { Excluded } \\
(n=151)\end{array}$ \\
\hline \multicolumn{3}{|l|}{ Gender } \\
\hline Male & $40.7 \%$ & $33.8 \%$ \\
\hline Female & $59.3 \%$ & $66.2 \%$ \\
\hline \multicolumn{3}{|l|}{ Age } \\
\hline $0-18$ yr & $28.6 \%$ & $25.8 \%$ \\
\hline $19-40 \mathrm{yr}$ & $46.2 \%$ & $51.7 \%$ \\
\hline $41-60 \mathrm{yr}$ & $15.2 \%$ & $15.2 \%$ \\
\hline$>60 \mathrm{yr}$ & $9.9 \%$ & $7.3 \%$ \\
\hline \multicolumn{3}{|l|}{ Race } \\
\hline White & $79.1 \%$ & $72.8 \%$ \\
\hline African American & $7.7 \%$ & $11.3 \%$ \\
\hline Asian & $6.6 \%$ & $2.0 \%$ \\
\hline Hispanic & $1.1 \%$ & $0.0 \%$ \\
\hline Other & $2.2 \%$ & $7.9 \%$ \\
\hline Unknown & $3.3 \%$ & $6.0 \%$ \\
\hline \multicolumn{3}{|l|}{ Injury severity score } \\
\hline 0 & $27.5 \%$ & $31.8 \%$ \\
\hline 1 & $42.9 \%$ & $44.4 \%$ \\
\hline 2 or more & $28.6 \%$ & $17.9 \%$ \\
\hline Unknown & $1.1 \%$ & $6.0 \%$ \\
\hline \multicolumn{3}{|l|}{ Discharge status } \\
\hline Admitted & $23.1 \%$ & $13.9 \%$ \\
\hline Discharged* & $75.8 \%$ & $86.1 \%$ \\
\hline
\end{tabular}

*One patient died in the emergency department.

ical injury severity was determined by one of the investigators (MAG) after reviewing the medical record, and the Abbreviated Injury Scale (AIS-90) ${ }^{2}$ was used to compute the Injury Severity Score (ISS) for each patient. ${ }^{3}$ The ISS scores were calculated by an individual who had received training in injury scoring.

Measurements. Crash characteristics were recorded using the variables of interest (Fig. 1). This form was modified from our previous study and used variables agreed upon by all study investigators. Variables of interest included restraint use (RU), airbag deployment (AD), and type of impact (TI). For TI, decision rules were drafted to facilitate coding. The first impact a vehicle sustained was recorded since this was always clearly indicated in the data sources. If a vehicle rollover occurred, this supervened because it was believed to be a significant crash event.

The reports were obtained by one of the investigators (MAG) and random numbers were assigned to each source. A master list with a unique identifier linked each data source to a particular crash. A second investigator (PWB) abstracted the randomized records for each data source to the study form. Each data source was provided to PWB separately for coding to prevent case identification. A third investigator (RJG) was provided a random sample of 20 reports to abstract from each data source to assess the interrater reliability of the abstraction process.

Data Analysis. Using the crash investigation as the standard comparison, concordance for each data source was calculated using kappa. Kappa was also calculated for the variables of interest. Percent agreement between the crash investigation and the variable options for each data source was also calculated. Interrater reliability of the abstraction process was measured by kappa.

\section{RESULTS}

Ninety-one patients were enrolled in the study. During the study period, 151 additional patients were identified for study enrollment but were not enrolled. Reasons for nonparticipation included: vehicle moved outside of study area by tow yard or insurance company (12\%), patient refused to participate (24\%), vehicle junked or repaired (29\%), or unable to contact patient due to inability to reach by telephone or incorrect telephone number from patient registration system $(35 \%)$. Despite the large number of patients who did not participate, Table 1 shows the nonparticipants and the participants to be very similar. While the nonparticipants were more often female and less frequently hospitalized, the two groups were similar in other characteristics. The median ISS was 1 for both participants and nonparticipants.

Table 2 shows agreement between the crash investigation and each variable by data source using kappa. Agreement with the crash investigation ranged from a high of 0.781 for type of impact on the PR to a low of 0.154 for airbag deployment on the AR. Agreement between the crash investiga-

TABLE 2. Agreement between Crash Investigation and Variable by Data Source*

\begin{tabular}{lcc}
\hline & $\begin{array}{c}\text { Agreement } \\
\text { (Kappa) }\end{array}$ & 95\% CI \\
\hline Restraint use & & \\
PR & 0.233 & $0.104,0.361$ \\
AR & 0.242 & $0.117,0.368$ \\
EDR & 0.242 & $0.107,0.378$ \\
Airbag deployment & & \\
PR & 0.611 & $0.440,0.782$ \\
AR & 0.154 & $0.070,0.238$ \\
EDR & 0.455 & $0.289,0.621$ \\
Type of impact & & \\
PR & & $0.679,0.883$ \\
AR & 0.781 & $0.429,0.667$ \\
EDR & 0.548 & $0.450,0.687$ \\
\hline
\end{tabular}

*PR = police report; $\mathrm{AR}=$ ambulance report $; \mathrm{EDR}=$ emergency department record. 
tion and each data source was $0.588(95 \%=\mathrm{CI}$ 0.508 to 0.667$)$ for the PR, $0.330(95 \% \mathrm{CI}=0.252$ to 0.407$)$ for the $\mathrm{AR}$, and $0.492(95 \% \mathrm{CI}=0.413$ to 0.572 ) for the EDR. Variable agreement was 0.239 $(95 \% \mathrm{CI}=0.164$ to 0.314$)$ for RU, $0.350(95 \% \mathrm{CI}=$ 0.268 to 0.432 ) for $\mathrm{AD}$, and 0.631 (95\% CI $=0.563$ to 0.698 ) for TI.

Table 3 depicts percent agreement for variable options with the crash investigation organized by data source. The crash investigator was most often in agreement with the data source options for the following: restrained occupants, deployed and nondeployed air bags, and most impact types, including frontal, rear end, and rollover. The crash investigator was most often in disagreement with the data source options for the following: unrestrained occupants, shoulder belt only occupants, and the remaining impact types, including driver side and passenger side impacts.

Table 4 shows the number of variables coded as unknown and the percentage of variables coded as unknown as compared with total disagreement between the crash investigation and variables. Overall, variables coded as unknown represented a large percentage of total disagreement for $\mathrm{AD}$ for all data sources and, in particular, the AR. Unknown was also coded quite frequently for TI on the AR and the EDR.

The kappas for interrater reliability for all data sources were above 0.80 for each data source. Kappa greater than 0.80 is considered excellent agreement. $^{4}$

\section{DISCUSSION}

The collection of crash characteristic data is important for both acute patient care and research. While some studies have questioned the usefulness of mechanism of injury to triage trauma patients appropriately ${ }^{5-8}$ the American College of Surgeons still includes mechanism of injury in its trauma protocol. ${ }^{9}$ Certain criteria (i.e., rollover and intrusion of opposite side compartment) have the ability to identify seriously injured patients. ${ }^{8}$ It is also known that safety belt use and the deployment of an air bag tend to lessen severity of injury. ${ }^{10-12}$ These protective devices can also cause injuries, especially if an air bag deploys when a patient is unrestrained. ${ }^{13,14}$ Similarly, the direction of impact can play a role in the injury pattern. ${ }^{15-17}$

Prior research has helped to determine how to effectively triage patients as well as how to efficiently allocate resources. The initial reporting of certain crash characteristics can influence the triage of trauma patients as well as deployment of resources required to care for them. To this end, at least one recent study questions the accuracy of
TABLE 3. Variable Option Agreement with Crash Investigation by Data Source*

\begin{tabular}{lrrrr}
\hline & & \multicolumn{3}{c}{$\%$ Agreement } \\
\cline { 3 - 5 } Variable Option $\dagger$ & $n$ & PR & AR & EDR \\
\hline Restraint use & & & & \\
$\quad$ Unrestrained & 41 & 0.292 & 0.317 & 0.341 \\
$\quad$ Restrained & 41 & 0.805 & 0.829 & 0.829 \\
Shoulder only & 6 & 0.167 & 0 & 0.167 \\
& & & & \\
Airbag deployment & & & & \\
$\quad$ Deployed & 20 & 0.800 & 0.500 & 0.600 \\
$\quad$ Not deployed & 70 & 0.900 & 0.357 & 0.857 \\
& & & & \\
Type of impact & & & & \\
$\quad$ Frontal & 32 & 0.906 & 0.813 & 0.719 \\
$\quad$ Driver side & 17 & 0.882 & 0.471 & 0.471 \\
Passenger side & 17 & 0.471 & 0.176 & 0.294 \\
Rear end & 10 & 0.900 & 0.900 & 0.900 \\
Rollover & 15 & 1.00 & 0.867 & 1.00 \\
\hline
\end{tabular}

$* \mathrm{PR}=$ police report; $\mathrm{AR}=$ ambulance report; $\mathrm{EDR}=$ emergency department record.

†Options with less than five responses were excluded.

TABLE 4. Unknown Data as a Percentage of Disagreement between Crash Investigation and Variable by Data Source

\begin{tabular}{lc}
\hline $\begin{array}{c}\text { Variable by Data } \\
\text { Source* }\end{array}$ & $\begin{array}{c}\text { Unknown } \dagger \\
\text { (Unknown \% of Disagreement) }\end{array}$ \\
\hline Restraint use & \\
PR & $7(15.9 \%)$ \\
AR & $10(23.3 \%)$ \\
EDR & $5(11.9 \%)$ \\
Airbag deployment & \\
PR & $6(50.0 \%)$ \\
AR & $54(96.4 \%)$ \\
EDR & $13(72.2 \%)$ \\
Type of impact & \\
PR & $0(-)$ \\
AR & $12(37.5 \%)$ \\
EDR & $12(38.7 \%)$ \\
\hline
\end{tabular}

*PR = police report; $\mathrm{AR}=$ ambulance report; $\mathrm{EDR}=$ emergency department record.

$\dagger$ Coded unknown after reviewing the PR, AR, and EDR.

retrospective trauma triage research. ${ }^{18}$ If crash characteristic data in trauma registries are inaccurate, this could have implications for studies conducted using this data. Researchers need to be aware of potential inaccuracies in data sources containing information on MVC crash characteristics.

Regarding the cause of discordance in the current study between the data sources and the independent crash investigation, there are a few possibilities. First, there was a relatively large amount of data that was missing upon review of the data sources. Had this information been reported, it is likely that agreement with the crash investigation would have been higher. Second, 
Michigan has a mandatory safety belt law. Patients often report that they were wearing a safety belt to a police officer and health care personnel to avoid a citation. Safety belt use was determined during the crash investigation using biomechanics and this often led to disagreement with data sources. Finally, side impacts were reported as frontal impacts in a number of cases. While police officers and paramedics at the scene may have believed they were correctly reporting a front impact, the crash investigation uses vector analysis to correctly determine impact. Often, offset frontal collisions are actually classified as side impacts with regard to the forces applied.

We believe our studies are unique in that the different data sources are compared with an independent crash investigation for accuracy. We were not able to find other studies using similar methods. However, there are studies that would seem to support the findings presented here. Hunt et al. have shown that ARs do not document the type of impact well. Up to $48 \%$ of reports could not document the area of impact and $61 \%$ of severity of impact. ${ }^{19}$ Santana and Martinez have shown that emergency physicians inaccurately describe crash characteristics up to $74 \%$ of the time when compared with PRs. ${ }^{20}$ While we cannot draw direct conclusions from a comparison of these studies, the overall accuracy rates of the PR being more accurate than the EDR, which in turn is more accurate than the AR, would seem to be in line with the findings from these other studies.

It is disturbing that none of the data sources studied seem to have a desirable level of accuracy. The PR could be considered reasonable were it not for the variable RU. The AR should not be considered accurate, at least for this population, due primarily to the large proportion of unknown data. It is possible that a different report form, as described in a study by Burstein et al., ${ }^{21}$ would make the AR a more accurate device. Methods to account for the potential inaccuracy of the data sources need to be developed.

\section{LIMITATIONS AND FUTURE QUESTIONS}

Although the method for enrolling patients was altered during the study, it was determined that this would not introduce any significant bias into the study. Since the study used only one crash investigator, there was concern that the crashes needed to be spaced apart to ensure that the investigator was not overburdened when the study began. However, it became necessary to alter the protocol for enrollment because not enough patients were being enrolled. Upon reviewing demographic differences between participants and nonparticipants, no significant difference was detected (Table 1). While the results are generalizable for the study population of interest, they may not be generalizable to another population.

Although the crash investigator is highly trained and knowledgeable, his investigations reflect the experience of one individual, and intrarater reliability is of some concern. In some instances, it was difficult for the investigator to determine what had occurred in the crash, perhaps leading to assumptions that may or may not have been accurate. The use of a single, experienced and highly trained investigator likely represents the most accurate and consistent method of retrospective crash investigation in the discipline of impact biomechanics.

Future studies should focus on two areas. Studying different populations and regions of the country to determine whether the results are generalizable would be informative. More importantly, it is necessary for researchers to develop mechanisms to compensate for the inaccuracies that are likely inherent in similar databases in other states.

\section{CONCLUSIONS}

A cross-sectional, observational study was conducted to study three data sources and their accuracy in recording MVC characteristics. Data from 91 MVCs were abstracted from the PR, AR, and EDR and compared with an independent crash investigation. Variables studied were $\mathrm{RU}, \mathrm{AD}$, and TI. Agreement using kappa between the crash investigation and each data source was 0.588 for the PR, 0.330 for the AR, and 0.490 for the EDR. This represents fair to moderate ${ }^{4}$ agreement between the data sources and the crash investigation. Determining the acceptable level of accuracy will be up to each researcher and could impact the data sources used. This study also raises questions about trauma registry data and other researchers that use these data sources. Further investigations into their accuracy and how improvements and/or compensation may be made with regard to accuracy are warranted.

The authors acknowledge the following individuals for their contribution to this study: Joel MacWilliams, University of Michigan Transportation Research Institute; Randy Bloom, Huron Valley Ambulance; and Mimi Leung, a graduate student at the University of Michigan School of Public Health when the study was conducted.

\section{References}

1. Grant RJ, Gregor MA, Maio RF, Huang SS. The accuracy of medical records and police reports in determining motor vehicle crash characteristics. Prehosp Emerg Care. 1998; 2: 23-8. 
2. Association for the Advancement of Automotive Medicine. The Abbreviated Injury Scale (AIS)-1990 Revision. Des Plaines, IL: AAAM, 1990.

3. Baker SP, O'Neill B, Haddon W Jr, Long WB. The Injury Severity Score: a method for describing patients with multiple injuries and evaluating emergency care. J Trauma. 1974; 14: 187-96.

4. Landis RJ, Koch GG. The measurement of observer agreement for categorical data. Biometrics. 1977; 33:159-74.

5. Simon BJ, Legere P, Emhoff T, Fiallo VM, Garb J. Vehicle trauma triage by mechanism: avoidance of the unproductive evaluation. J Trauma. 1994; 37:645-9.

6. Esposito TJ, Offner PJ, Jurkovich GJ, Griffith J, Maier RV. Do prehospital trauma center triage criteria identify major trauma victims? Arch Surg. 1995; 130:171-6.

7. Henry MC, Alicandro JM, Hollander JE, Cassara G, Thode HC Jr. Evaluation of American College of Surgeons trauma triage criteria in a suburban and rural setting. Am J Emerg Med. 1996; 14:124-9.

8. Henry MC, Hollander JE, Alicandro JM, Cassara G, O'Malley S, Thode HC Jr. Incremental benefit of individua American College of Surgeons trauma triage criteria. Acad Emerg Med. 1996; 3:992-1000.

9. American College of Surgeons, Committee on Trauma. Resources for optimal care of the injured patient: 1999. Chicago: ACS, 1998.

10. Viano DC. Restraint effectiveness, availability and use in fatal crashes: implications to injury control. J Trauma. 1995; 38:538-46.

11. Kuner EH, Schlickewei W, Oltmanns D. Injury reduction by the airbag in accidents. Injury. 1996; 27:185-8.

12. Henry MC, Hollander JE, Alicandro JM, Cassara G, O'Malley S, Thode HC Jr. Prospective countywide evaluation of the effects of motor vehicle safety device use on hospital resource use and injury severity. Ann Emerg Med. 1996; 28:
627-33.

13. Sutyak JP, Vikas P, Jammond JS. Air bags alone compared with the combination of mechanical restraints and air bags: implications for the emergency evaluation of crash victims. South Med J. 1997; 90:915-9.

14. Porter RS, Zhoa N. Patterns of injury in belted and unbelted individuals presenting to a trauma center after motor vehicle crash: seat belt syndrome revisited. Ann Emerg Med. 1999; 32:418-24.

15. Loo GT, Siegel JH, Dischinger PC, et al. Airbag protection versus compartment intrusion effect determines the pattern of injuries in multiple trauma motor vehicle crashes. J Trauma. 1996; 41:935-51.

16. McLellan BA, Bizoli SB, Brenneman FD, Boulanger BR, Sharkey PW, Szalai JP. Injury pattern and severity in lateral motor vehicle collisions: a Canadian experience. J Trauma. 1996; 41:708-13.

17. Dischinger PC, Cushing BM, Kerns TJ. Injury patterns associated with direction impact: drivers admitted to trauma centers. J Trauma. 1993; 35:454-8.

18. Jones SE, Brenneis A. Study design in prehospital trauma advanced life support-basic life support research: a critical review. Ann Emerg Med. 1991; 20:857-60.

19. Hunt RC, Brown RL, Cline KA, Krohmer JR, McCabe JB, Whitley TW. Comparison of motor vehicle damage documentation in emergency medical services run reports compared with photographic documentation. Ann Emerg Med. 1993; 22: 651-6.

20. Santana JR, Martinez R. Accuracy of emergency physician data collection in automobile collisions. Proc Assoc Adv Automot Med. 1993; 37:263-9.

21. Burstein JL, Henry MC, Alicandro JM, McFadden K, Thode HC Jr, Hollander JE. Evidence for and impact of selective reporting of trauma triage mechanism criteria. Acad Emerg Med. 1996; 3:1011-5.

\section{TEAChing PROFESSIONALISM}

Professionalism cases are available on the SAEM website, www.saem.org. These items are meant to serve as a starting point for discussion surrounding proper behavior and proper action. While easy answers are often elusive, these cases can promote provocative discussion and serve as important reminders that our overriding responsibility is to serve the patient's interests. 